\title{
Case Report: Mediastinal Mass in an Infant - A Source of Concern?
}

\author{
Palanikumar Balasundaram, Shrestha Banga, Mohd Hamza and Ratna B Basak*
}

Brookdale Hospital Medical Center, Brooklyn, NY, US

"Corresponding author: Basak RB, MD, Department of Pediatrics, Brookdale Hospital Medical Center, Brooklyn, NY, US, Tel: +88 01747439378; E-mail: ratnabimalbasak@gmail.com

Received: December 15, 2018; Accepted: December 27, 2018; Published: December 30, 2018

\section{Introduction}

The discovery of a mediastinal mass is usually alarming. A benign organ, thymus is a bi-lobed encapsulated structure located in the anterior mediastinum, anterior to the great vessels. It is usually prominent in young children with the classical morphological appearance of a nautical sail, confirming its normal position [1-3]. We present a child with an x-ray of a thymus mimicking a mediastinal mass which was located in the inferior aspect of the heart.

\section{Case}

A 6-month-old, previously healthy infant presented with a one week history of cough, runny nose, and one day history fever and rapid breathing. His vitals were: HR 120/M, RR 45/m, Sat of 95\% in room air. Physical examination revealed an active boy, in mild respiratory distress, with normal breath and heart sounds. Labs showed a normal CBC, BMP, and urinalysis. RSV and rapid flu antigen were negative. X-ray chest in the ED revealed no evidence of pneumonia but had an enlarged cardiac silhouette (FIG. 1, A and B.). For this finding, ECHO was done which revealed normal findings except for a tiny PFO. He was provisionally diagnosed as Bronchiolitis and was treated symptomatically with intravenous fluids, oxygen, saline nebulization and bulb suction. A repeat $x$-ray chest done on day 3 of admission revealed the same findings. (FIG. 2, A and B) CT scan of chest was done to rule out anterior mediastinal mass which revealed a thymus extending along the anterior mediastinum draping over the inferior aspect of the heart (FIG. 3).

\section{Discussion}

The Thymus is a pyramid-shaped lymphoid organ that plays a vital role in the development and maturation of the immune system in childhood by B and T cells [2]. It is located in the anterior mediastinum, overlying the pericardium, aortic arch, innominate vein and trachea. It can extend superiorly to the lower pole of the thyroid and inferiorly to the diaphragm [3]. Cross sectional imaging shows that the thymus is a homogenous soft - tissue structure without calcification or compression on the airways or vascular structure [4]. Unlike other lymphoid structures, it grows rapidly and attains its greatest size in the first few months of life and does not grow any larger beyond puberty [2,4]. It is then replaced by fat and fibrous tissue. In the

Citation: Basak RB, Balasundaram P, Banga S, et al. Case Report: Mediastinal Mass in an Infant - A Source of Concern? Clin Case Rep Open Access. 2018;1(2):116. 
neonatal period, the normal thymic shadow should be twice the width of the third dorsal vertebrae [2]. Thymus can involute in size in response to stress like in severe sepsis, major surgery, and with immunosuppressant's which is transient and usually returns to normal or even 50\% larger after the stress resolves: known as rebound thymic hyperplasia [2-4]. In a child below 3 years of age, it appears as a soft tissue density with smooth borders, superimposed on cardiac shadows, usually as a supra cardiac or retrosternal opacity. On CT, normal pediatric thymus often appears as a generous anterior mediastinal soft tissue draping over the superior aspect of the heart with a quadrilateral morphology in the coronal plane with convex margins, anterior to the proximal ascending aorta, pulmonary outflow tract and distal to the superior vena cava [4]. With increasing age, it becomes triangular in shape with straight or concave margins [2]. In our patient, the thymus was draping over the inferior aspect of heart which is an unusual location.

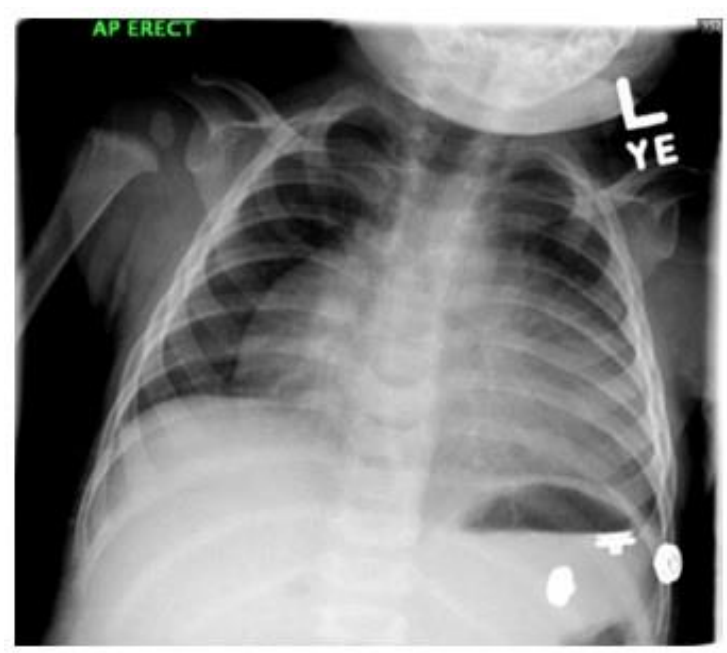

FIG. 1. A and B. CXR Frontal and lateral views of the chest. The cardiac silhouette is markedly enlarged. The lungs are clear with no pulmonary consolidation or CHF. There is no pleural fluid or pneumothorax.

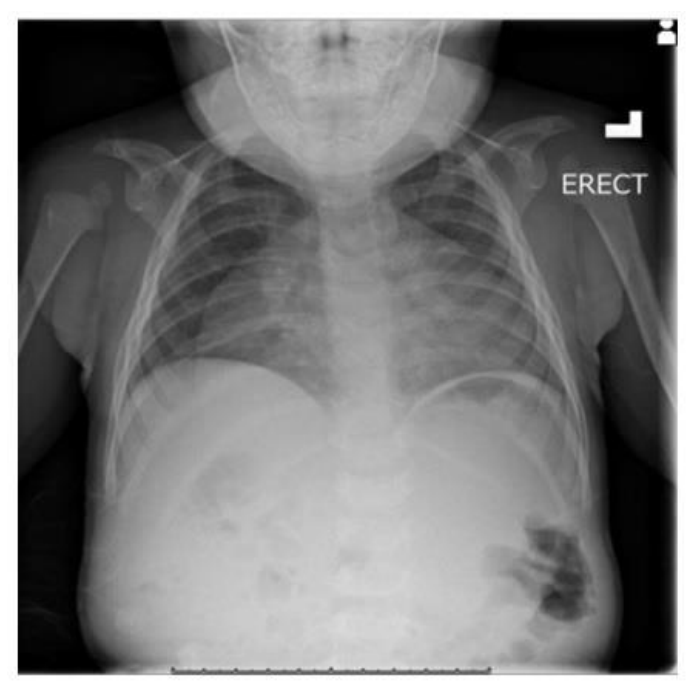

FIG. 2. A and B. CXR Frontal and lateral views of the chest. The cardiac silhouette is enlarged. The lungs are clear with no pulmonary consolidation or CHF. There is no pleural fluid or pneumothorax. No interval change noted from prior x-ray. 

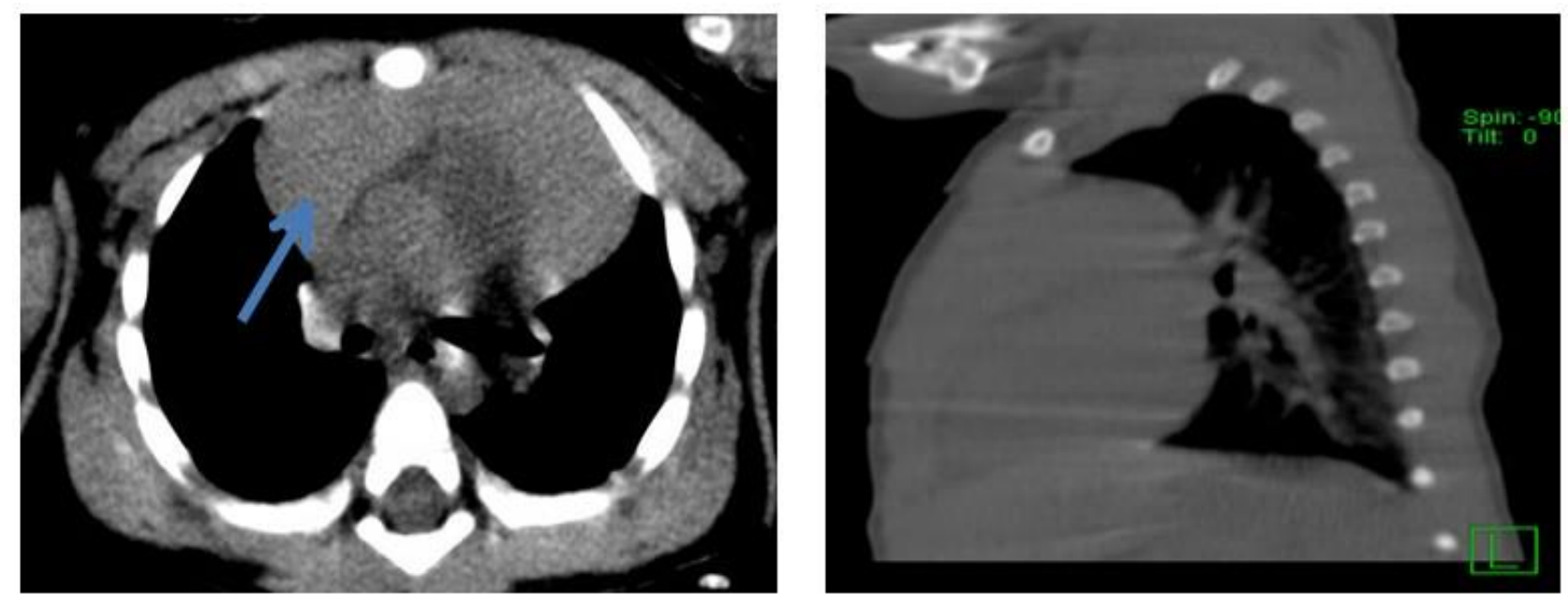

FIG. 3. CT scan of chest. Lungs: There are mild hypoventilatory changes within the bilateral lungs. There is no mass or consolidative process. Pleural space: Normal. No pneumothorax. No pleural effusion. Heart: The heart is normal in size. Cardiac chambers are unremarkable in distribution. Mediastinum: The thymus extends along the anterior mediastinum to drape over the inferior aspect of the heart. There is no discrete mediastinal mass.

\section{Conclusion}

The thymus can be located anywhere from the lower pole of thyroid to the diaphragm. It can have a variety of shape and size even in the same individual. It gradually involutes with age and may acutely shrink during periods of stress and during the recovery phase, but it may grow back to its original size or even larger. These anatomic variations in positions and dynamic changes appear to be the main source of confusion with pathological conditions [2].

\section{Conflict of Interest}

None

\section{REFERENCE}

1. Lee EY, Miller FH. Practical Pediatric Imaging. Radiol Clin North Am. 2017;55(4):609-904.

2. Nasseri F, Iftekhari F. Clinical and Radiologic Review of the Normal and Abnormal Thymus: Pearls and Pitfalls. Radiographics. 2010;30(2):413-28.

3. Ranganath SH, Lee E, Restrepo R, et al. Mediastinal masses in children. Am J Roentgenol. 2012;198(3):W197W216.

4. Manchanda S, Bhalla A, Jana M, et al. Imaging of pediatric thymus: Clinicoradiological approach. World J Clin Pediatr. 2017;6(1):10-23. 